DOI 10.19195/2084-2546.27.5

\author{
VALENTYNA KOTENKO \\ ORCID: 0000-0003-3934-3871 \\ Universidad Nacional de Kiev Tarás Shevchenko \\ Correo: valya.kotenko@gmail.com
}

\title{
Correr la lengua: el fenómeno de la Korrika
}

Palabras clave: lenguas minorizadas - discurso de deporte - euskera Korrika - lema.

\section{Introducción}

En este estudio se propone prestar atención al vínculo que existe entre el deporte - debido a que es una manifestación de la práctica social y una característica intrínseca de la sociedad española - y la lengua en el marco del discurso de deporte de España. En particular, intentamos hacer un esbozo sobre la Korrika, una carrera organizada cada dos años en la Comunidad Autónoma Vasca a favor del idioma vasco - el euskera - y destacar sus funciones pragmáticas.

Tal y como destacan los lingüistas, el análisis del discurso es la disciplina que, en lugar de proceder a un análisis lingüístico del texto en sí mismo, o a un análisis sociológico o psicológico de su contexto, tiene como objetivo articular su enunciación con un determinado lugar social ${ }^{1}$. El análisis del discurso se presenta como la disciplina que usa unos símbolos lingüísticos en un contexto cultural concreto $^{2}$, que estudia el lenguaje para fines sociales, expresivos y referenciales ${ }^{3}$. El núcleo del discurso del deporte es una serie de actividades deportivas, relacionadas entre sí en forma de competiciones y que son institucionales ${ }^{4}$. El deporte es, según S. Connor, una de las modali-

${ }^{1}$ D. Maingueneau, Términos clave para el análisis del discurso, Buenos Aires, Nueva Visión, 1999, p. 17.

${ }^{2}$ Shi-xu, A cultural approach to discourse, New York, Palgrave Macmillan, 2005, p. 19.

${ }^{3}$ P. Charaudeau, D. Maingueneau, Diccionario de análisis de discurso, Buenos Aires-Madrid, Amorrortu SL, 2005, p. 33.

${ }^{4}$ P. Parbelas, Elementos de la sociología del deporte, Málaga, Instituto Andaluz del Deporte, 2003, p. 74. 
dades de la actividad social ${ }^{5}$. Las prácticas deportivas en la sociedad española - tanto en su cultura lúdica como desde el enfoque histórico y político- son mucho más que una actividad física. Su destacado papel consiste también en la cristalización de los conceptos de la vida actual plasmados en el discurso deportivo, que es un metadiscurso por su naturaleza.

La mayor dificultad a la hora de trabajar con el tema de la Korrika consiste en la escasa bibliografía, ya que en español se publicó solo un libro de Teresa del Valle, Korrika. Rituales de lengua en el espacio (1988), por lo cual para componer el corpus del estudio hemos acudido también a los lemas de las pancartas, las letras de canciones y los artículos de prensa. La metodología se basa en el análisis del discurso del deporte (práctica discursiva), la descripción lingüística y el análisis pragmático.

Si estudiamos el deporte como un fenómeno social, podemos descubrir que junto al protagonismo del fútbol - que se ha convertido en casi una religión para miles de personas en toda la Península y que además es el espectáculo deportivo que moviliza el mayor volumen de recursos económicos y publicitarios de todo el deporte español ${ }^{6}$ - existen otras herramientas del ámbito deportivo para conseguir objetivos de gran calado social, ya que tanto el lenguaje como el deporte son fenómenos sociales. La Korrika es una carrera que se prolonga ininterrumpidamente, día y noche sin parar, y que también tiene las características de una ceremonia deportiva surgida de un movimiento popular, pero que hace énfasis en la importancia del idioma. Korrika es una iniciativa popular proeuskera, una carrera durante la cual se recorre el territorio de Euskal Herria - los territorios de las siete provincias vascófonas de Francia y España - con el único fin de apoyar el desarrollo de este idioma, popularizarlo y a la vez - rendirle homenaje. Se puede afirmar que este evento está contextualizado, es interactivo y lo estudiamos desde el punto de vista pragmático como un uso lingüístico contextualizado.

El euskera es una lengua, una cultura, una vida: el idioma de nuestra tierra, nuestro bagaje cultural y un modo de ser y vivir en el mundo. Hace años que las personas vascoparlantes y las vascófilas emprendimos la tarea de fortalecer nuestra lengua, anhelando un futuro mejor. $\mathrm{Tu}$ lengua, tu cultura, tu mundo. Korrika 20 te habla a ti y quiere hablar contigo, para que conozcas el euskera y lo utilicemos [...] Entendemos que es necesario cuidar y proteger el euskera. El euskera debe desarrollarse en igualdad de condiciones, día a día y en todos los ámbitos, para cada ciudadano y ciudadana pueda vivir en igualdad de condiciones, día a día y en todo lugar. Únete. ¡Suma! Yo, tú, él, ella, nosotros, vosotras, ellos. [...] Es importante unirnos y aportar: mi lengua, tu lengua, nuestra cultura, nuestras culturas, este mundo, estos mundos. Al fin y al cabo, apoyar a un idioma es actuar en favor del conjunto de las lenguas; en favor de la cultura universal. Te invitamos a la fiesta por la igualdad de las lenguas ${ }^{7}$.

${ }^{5}$ S. Connor, A Philosophy of Sport, London, Reaktion Books Ltd., 2011, p. 12.

${ }^{6}$ M.G. Ferrando, Postmodernidad y Deporte: entre la individualización y la masificación. Encuesta sobre hábitos deportivos de los españoles, Madrid, CIS (Centro de Investigaciones Sociológicas), 2006, p. 24.

${ }^{7}$ Folleto de la Korrika 20 (2017), en: Página oficial de la Korrika, <http://www.korrika.eus/ cas/quees korrika/mensaje.html>, 27 de abril de 2018. 
Son las palabras del lema de la última edición de la Korrika 2017, en los cuales se pueden observar los conceptos clave que sirven como impulso para hablar del tema de la relación entre una actividad deportiva y a la vez lingüística en el contexto del euskera: lenguas minorizadas, riqueza lingüística, fortalecimiento y protección de la lengua, igualdad de las lenguas. El uso de los deícticos hace un llamamiento a cada lector $\mathrm{u}$ oyente de dicho enunciado y los anima a participar en este evento.

\section{Lengua minoritaria frente a lengua minorizada: el caso vasco}

Según la Constitución Española de 1978 se reconoce el carácter multilingüe y multicultural en el país. De este modo, los artículos 3 (p. 2, 3), 20 (p. 3) y el 148 (p. 17) garantizan el derecho de usar las lenguas de las comunidades autónomas respectivas (el catalán, vasco y gallego) ${ }^{8}$. Según los datos de la encuesta sociolingüística del año 2016, en Euskadi unas 631.000 personas son vascohablantes (el 33,9\% de la población mayor de 16 años) y 356.000 personas son vascohablantes pasivos (el 19,1\%). Según la Carta europea de las lenguas regionales o minoritarias, por "lengua regional o minoritaria" se entienden las lenguas que son tradicionalmente practicadas dentro de un territorio estatal formando un grupo numéricamente más reducido que el resto de la población del Estado; que son diferentes de la lengua oficial del Estado; sin incluir otros dialectos de lenguas oficiales del Estado o lenguas de emigrantes ${ }^{9}$. Sin embargo, existe una cierta diferencia entre las lenguas minoritarias y minorizadas:

La lengua vasca constituye en la actualidad el instrumento cultural más representativo de la comunidad que la ha usado y conservado, poseyendo las peculiaridades de naturaleza sociopolítica, como son también su carácter de lengua minoritaria (es utilizada por un pequeño número de hablantes) y, al mismo tiempo, también minorizada, ya que en diferentes circunstancias históricas, antiguas y recientes, el uso de euskera estuvo postergado y, en algunas ocasiones, prohibido y relegado a funciones de poco prestigio sociocultural ${ }^{10}$.

Hay que subrayar qué es exactamente el término "minorizada", que se usa a la hora de hablar de la Korrika por parte de sus organizadores y que encontramos en sus folletos. Tal y como lo afirma F. Zubiaga, toda la historia del País Vasco en el contexto de España o de Francia es la lucha por el olvido

${ }^{8}$ Constitución Española del 1978, en: Agencia Estatal Boletín Oficial del Estado, <https:// www.boe.es/buscar/doc.php?id=BOE-A-1978-31229>, 27 de abril de 2018.

${ }^{9}$ Council of Europe, European Charter for Regional or Minority Languages (Spanish Version), Strasbourg, 5.11.1992, parte I, art. 1, https://www.coe.int/t/dg4/education/minlang/textcharter/Charter/ Charter es.pdf $>, 27$ de abril de 2018.

${ }^{10}$ M.A. Etxebarria, La diversidad de las lenguas en España, S.L.U. Espasa Libros, 2002, p. 211. 
o la memoria del vascuence, la lengua más antigua de Europa ${ }^{11}$. La situación actual efectivamente va cambiando, ya que el vasco está presente en los medios de comunicación y en el proceso educativo de esta comunidad autónoma. Al mismo tiempo vale la pena afirmar que cuando el uso de un idioma está restringido y delimitado dentro de un área geográfica, y por el mero hecho de tener un número de hablantes bastante escaso en una situación de cierta diglosia, de una $u$ otra forma la lengua se encuentra en una situación de peligro.

Según el atlas lingüístico vasco, el euskera es un hecho social minoritario y geográficamente fragmentado ${ }^{12}$. El caso de esta lengua siempre ha sido un punto de referencia significativo en el contexto de la expresión de la identidad del pueblo vasco. De esta manera, la Korrika, como carrera en favor del euskera, es una herramienta que permite, antes de todo, manifestar la existencia de esta lengua recorriendo y físicamente perfilando el territorio geográfico de la difusión de este idioma por las comunidades autónomas que históricamente lo hablaron.

Por otro lado, como el euskera es un idioma con una tradición literaria bastante tardía (1545), la tradición de enseñar y transmitir el idioma de una forma oral siempre ha desempeñado un papel muy importante. Según T. del Valle, la Korrika ocurre en el tiempo (en el sentido histórico), en el territorio (en la gente que ocupa un espacio concreto), en las distintas formas de vida conectadas con diversidades ecológicas y económicas, en la transmisión natural (en las generaciones), en el espacio privado, público, medios de comunicación; asimismo evoca lo arcaico, lo más tradicional, lo ancestral, lo idílico que se proyecta al mismo tiempo hacia el porvenir ${ }^{13}$, así que podemos afirmar que la organización de la Korrika se impregna del concepto propio de la oralidad.

\section{La Korrika y la verbalización en espacio}

La Korrika nace en 1980, en un periodo cuando se pasa de un deporte "ideologizado" y con fines políticos (nacionalismo deportivo, adoctrinamiento de la juventud, etc.) a un deporte que "promueve y cimienta" una nueva nación española que se encuentra en continuo desarrollo a través de sus instituciones democráticas ${ }^{14}$. La carrera está organizada y patrocinada por AEK (Alfabetatze Euskalduntze Koordinakundea, Coordinadora de Alfabetización y Euskaldunización) y apoyada por HABE (Helduen Alfabetatze eta Ber-

${ }^{11}$ F. Inco Zubiaga, Vascuence, inconsciente colectivo, Erroteta, 2005, p. 99.

12 J.I. Ruiz Olabuénaga, Atlas lingüístico vasco, Gobierno vasco. Presidencia, Gabinete de prospección sociológica, 1984 , p. 1.

13 T. del Valle, Korrika: rituales de la lengua en el espacio, Barcelona, Anthropos, 1988, p. 293.

14 X. Pujadas, Atletas y ciudadanos. Historia social del deporte en España 1870-2010, Madrid, Alianza Editorial, 2011, p. 436. 
reuskalduntzerako Erakundea, Organización para la Alfabetización y la Reeuskaldunización de Adultos), por el Gobierno Vasco y la Euskaltzaindia (Academia de la Lengua Vasca). Tiene como objetivo impulsar la concienciación a favor del euskera y como máxima la revitalización de la lengua vasca en toda la Comunidad Autónoma Vasca. Además - como se indica en la página web oficial de la carrera - se trata de garantizar el derecho de estudiar gratuitamente euskera en toda Euskal Herria a los no-vascoparlantes. De esta manera se trata de dar un impulso al aprendizaje del euskera y promoverlo en un ambiente euskaldun. Otro objetivo de carácter más práctico es recabar fondos para llevar a cabo la enseñanza del euskera día a día en los centros del aprendizaje de $\mathrm{AEK}^{15}$, así como dirigir la atención al problema del apoyo de euskera, lo que podemos leer en las pancartas llevadas durante la carrera: Ertzainaren dirua euskara ikasteko (El dinero de la ertzaina para aprender euskara) ${ }^{16}$. En los últimos 37 años se han realizado 20 ediciones. Durante unos 10 días se recorre el territorio de las siete provincias sin detenerse en ningún momento con la frase Korrika ez da gelditzen (la Korrika no se para), que se repite durante la carrera. Tal y como lo destacó R. Barthes en la descripción de importancia del ciclismo en Francia durante el Tour, los franceses tienen cada año la oportunidad de conocer la longitud de las costas y la altura de sus montañas. Cada año una persona recompone la unidad material de su país, cada año recorre sus fronteras y sus productos ${ }^{17}$. Esta idea es también totalmente cierta en el contexto de la Korrika: superando los obstáculos naturales del paisaje local (valles, montes y agua) los corredores demuestran su vínculo con el territorio con sus propios pies y su esfuerzo físico. A diferencia de los deportes tradicionales, no se trata de ganar una competición o vencer a un rival, sino más bien apoyar una causa justa; aportando también con una finalidad formativa (el deporte como un conocimiento aplicado no conscien$t^{18}{ }^{18}$. Recorriendo el territorio de habla vasca los participantes demuestran su capacidad de sacar el euskera de los colegios y academias a la calle y - como lo designa T. del Valle - revitalizar el uso de la lengua, demostrar su vínculo con toda Euskal Herria ${ }^{19}$.

Cada edición tiene un lema particular y original en la que se enfatiza la importancia de la lengua vasca durante el acontecimiento deportivo: Herri bat gara, hizkuntza bat dugu - Somos un pueblo, tenemos una lengua:

1. 29.11-7.12.1980, Zuk ere esan bai euskarari - Tú también di "sí" al eukera;

2. 22.05-30.05.1982, AEK, euskararen alternatiba herritarra eta Korrika, herriaren erantzuna euskararen alde - AEK - alternativa popular del euskera y Korrika - la respuesta del pueblo en favor del euskera;

\footnotetext{
${ }^{15}$ Folleto de la Korrika 20 (2017), op. cit.

16 T. del Valle, op. cit., p. 190

${ }^{17}$ R. Barthes, What is Sport?, New Haven, Yale University Press, 2007, p. 29.

18 X. Pujadas, op. cit., p. 479.

19 T. del Valle, op. cit., p. 269.
} 
3. 3.12-11.12.1983, Euskaraz eta kitto! - iEuskara y basta!;

4. 31.05-9.06.1985, Herri bat, hizkuntza bat! - Un pueblo, una lengua;

5. 3.04-12.04.1987, Euskara, zeurea - Euskera tuyo;

6. 14.04-23.04.1989, Euskara Korrika eta kitto eta Euskal Herriak AEK - Euskera, Korrika y listo! Euskal Herria apuesta por la AEK;

7. 15.03-24.03.1991, Korrika euskara, euskaraz Euskal Herria - Corriendo en euskera, Euskal Herria en euskera;

8. 26.03-4.04.1993, Denok maite dugu gure herria euskaraz - Todos queremos a nuestro pueblo en euskera;

9. 17.03-26.03.1995, Jalgi hadi euskaraz - Sal en euskera;

10. 14.03-23.03.1997, Euskal Herria Korrika! - Euskal Herria corriendo!;

11. 19.03-28.03.1999, Zu eta ni euskaraz - Tú y yo en euskera;

12. 29.03-8.04.2001, Mundu bat euskarara bildu - Un mundo unido en euskera; futuro;

13. 4.04-13.04.2003, Herri bat geroa lantzen - Un pueblo trabajando el

14. 10.03-20.03.2005, Euskal Herria euskalduntzen. Ni ere bai! - Euskaldunizando Euskal Herria. ¡Yo también!;

15. 22.03-1.04.2007, Heldu hitzari, lekukoari, elkarlanari, euskarari, herriari - Agárrate a la palabra, al testigo, al trabajo en equipo, al euskera, al pueblo;

16. 26.03-5.04.2009, Ongi etorri euskaraz bizi nahi dugunon herrira! Bienvenido al pueblo que quiere vivir en euskera;

17. 7.04-17.04.2011, Maitatu, ikasi, ari... Euskalakari-Quiere, aprende, trabaja.... Euskalakari es una palabra inventada cuyo significado gira en torno al hablar - "el que habla";

18. 14.03-4.03.2013, Eman Euskara Elkarri - Dar euskera unos a otros;

19. 19.03-29.03.2015, Euskahaldun! Es una palabra compuesta que presenta el juego de palabras: euskaldun es una persona que habla euskera y ahaldun, empoderado; de modo que se podría traducir como empoderado con el euskera;

20. 30.03-9.04.2017, Batzuk - varios. Puede ser que aquí han separado "bat" (uno) y "zuk" (tú), para dar a entender algo así como "tú eres uno con nosotros".

Otras herramientas usadas en la Korrika para verbalizar el euskera en la calle y recalcar el eje lingüístico de este evento son las pancartas y carteles anunciadores, chapas, dorsales y pegatinas que lleva la gente durante la carrera. Según la idea establecida por la AEK, el enfoque lingüístico es el único que tiene importancia y la carrera como tal no persigue ningún otro objetivo. Sin embargo, los participantes aprovechan este evento masivo para abordar una variedad de temas en los eslóganes, que son muy variados desde la época de la Transición y hasta hoy en día.

Ante todo, por supuesto, son las pancartas a favor del euskera, las que nos sirven de material de estudio del discurso de deporte: Ez diguzue ken- 
duko gure hizkuntza - No nos vais a quitar nuestra lengua; Euskara benetan gure udaletan - El euskera de verdad, en nuestros Ayuntamientos; Nunahi eta noiznahi euskaraz - En cualquier parte y en cualquier momento en euskara ${ }^{20}$; Euskal herrian euskaraz - En Euskal Herria en euskera; Euskera OFIZIALA Euskera oficial $^{21}$, Herria sortzen EUSKARATIK eta EUSKARAZ - Haciendo el pueblo desde el euskera y en euskera ${ }^{22}$. El enfoque educativo es uno de los temas presentes en las pancartas durante la carrera. Una pancarta que dice Egunero goaz Amurriora euskara ikasteko, zergaitik ezin dugu Ordunan ikasi? - Cada día vamos a Amurrio a aprender euskara, ¿por qué no podemos aprenderlo en Orduña? o Ikastola Herri eskola - La escuela en euskera la escuela del pueblo; Euskaraz bizi nahi dut - Quiero vivir en euskera ${ }^{23}$, presta atención a un problema existente del aprendizaje de euskera en los colegios; otro, Hitz gutxiago eta diru gehiago. Hernaniko AEK - Menos palabras y más dinero. AEK en Hernani, destaca la importancia de AEK. Se trata tanto del papel de ikastolas como de planteamientos personales respecto a dicho tema. El desarrollo de las ikastolas también es uno de sus objetivos: Ikastola gure eskola - La ikastola nuestra escuela; Ikastola Herri escola - La ikastola la escuela del pueblo; Euskalherrian euskaraz - En Euskal Herria en euskera; Euskaraz bizi behar dut - Tengo que vivir en Euskara; Euskal escola Publiko Bakarra - Una única red de escuela pública vasca; Haurtzako ikastola - La guardería-ikastola) ${ }^{24}$.

El tema de las cuestiones políticas (Independentzia); presos políticos (No a la nuclerización, sí a la amnistia, Los refugiados asesinados también están en Korrika, Es gaude denak, presoak falta dira - No estamos todos, faltan los presos; Korrika bai, amnistía ere bai - Sí a la Korrika y a la amnistía también) ${ }^{25}$ ya ocupan mucho menos espacio en la realización de la Korrika, y - volveremos a subrayar - no son en ninguna manera el objetivo de la carrera. Sin embargo, existen casos en los que el discurso deportivo sintoniza con el discurso político y se hace referencia a unos acontecimientos pasados recientemente, por ejemplo, con la reivindicación respecto a los presos. Por ejemplo, durante la carrera pidieron la absolución de los encausados por la violencia callejera en Pamplona: Absoluzioa! ${ }^{26}$. La reivindicación laboral, el problema del paro o libertad de prensa y de radio, el uso del euskera en los juzgados también están en minoría temática: Epaitegietan Euskaraz - En los juzgados en euskera; Gure eskubide delako. Abokatu "BAT ZUK" Korrikaren alde - Porque es nuestro derecho. Abogados unidos en favor de euskera.

\footnotetext{
20 T. del Valle, op. cit., pp. 216 y 269.

21 Pancarta de la edición 20 en Navarra, 2017.

22 Pancarta de la edición 19 en Getxo, 2015.

23 T. del Valle, op. cit., p. 122.

24 Ibidem, pp. 210-212.

25 Ibidem, pp. 188 y 236.

26 Pancarta de la edición 20 en Navarra, op. cit.
} 
Durante los años 80 incluso aparecía la definición del papel de las mujeres: Emakumeak ere euskararen alde - Las mujeres también en favor del euskara. Uno de los casos muy curiosos es el apoyo de los extranjeros: Ni kanpotarra naiz izatez, baina Euskaldin bizi naiz, horregatik euskara ikasten ari naiz Korrikan - Soy forastero, pero vivo en Euskadi, por eso estoy aprendiendo euskera corriendo ${ }^{27}$. También hay pancartas con la denominación institucional de la Korrika: Euskararen Plangintza orain. Euskarazko Kulturaren Batzarra - La planificación del euskara ahora. Asamblea Cultural del Euskara.

En relación con el espacio, ya hemos mencionado las siete provincias de Euskal Herria, pues se trata de poder recorrer las provincias históricas sin dar importancia primordial a las fronteras administrativas actuales. Así, la última Korrika en marzo de 2017 recorrió durante 11 días más de 2535 kilómetros atravesando más de 200 localidades con la participación de cientos de miles de personas en Euskal Herria. Se puede apreciar como va recogiendo las variedades lingüísticas de los lugares por donde pasa (se trata de los dialectos del euskera) y refleja a su vez ciertas características de la situación de la lengua en aquellos territorios: Trebiño euskararen alde, Gu euskaldunak gara - Trebiño a favor del euskara, Nosotros somos euskaldunes ${ }^{28}$. Actualmente no hace distinción entre los territorios que son vascohablantes en su mayoría o no, por lo cual se organiza también, por ejemplo, en la Ribera de Navarra, Vitoria o Bilbao donde la mayoría de la gente no es vascohablante.

En su dimensión global es una fiesta que no solo consta de una carrera, sino que también abarca eventos culturales: canciones, danza, representaciones teatrales, concursos; todo organizado en euskera con el mismo fin de promover y popularizar el idioma, animar a la gente a usarlo demostrando su utilidad en la vida real. Lo que más nos llama la atención es que muchos de estos eventos son iniciativas de cada barrio, son totalmente voluntarios, sin ningún tipo de apoyo central de los organizadores. Este hecho demuestra que el evento tiene su repercusión en la sociedad y la empuja hacia la organización voluntaria del ambiente que acompaña a la carrera durante los días que dura. Se puede disfrutar de txarangas (grupo de músicos en la calle) y fanfarrias, bertsolariak (bertsolari es la persona que improvisa versos), kalejirak (fiestas de baile en las calles), txoznak (celebraciones asociadas con la calle o una plaza concreta, las casetas donde se despachan bocadillos y bebidas mientras suena música para que la gente disfrute del ambiente y baile), txistulariak (actuaciones musicales con tambores y txistu, la típica flauta vasca de tres agujeros) y actuaciones de trikitixa (grupo musicales que actúa en fiestas populares del País Vasco y cuya peculiaridad es el uso de un acordeón vasco y pandereta). Gracias a la producción teatral, proyección de videos y dibujos animados se establece la presencia de la lengua en la calle durante todos los días de duración. Por otro lado, es importante destacar que en los últimos años se han

\footnotetext{
27 T. del Valle, op. cit., p. 213.

${ }^{28}$ Ibidem, p. 174.
} 
creado ayudas y una serie de becas de creación desde AEK para promoverla. Se trata de Korrika Kulturala - conciertos, teatro, bertsos y danza ${ }^{29}$ - donde los grupos artísticos escogidos ofrecen sus actuaciones en distintos lugares.

Hablando de la presencia del arte en la Korrika podemos también destacar festivales, acciones culturales y la canción oficial. Durante toda la carrera suena esta canción que se compone para cada edición nueva. La canción de la edición 2017 se llama Zirkorrika ${ }^{30}$ y en español la primera parte dice así:

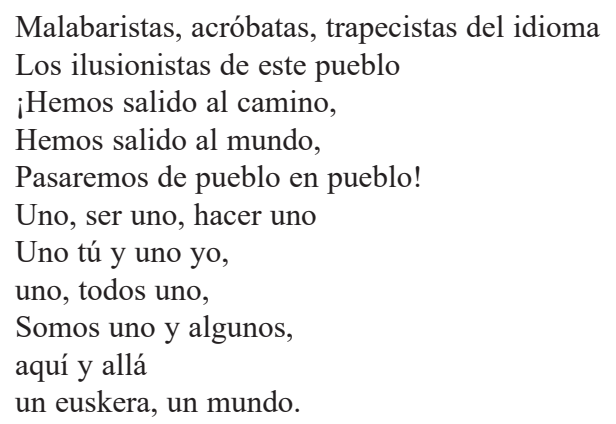

En este caso podemos observar de nuevo la abundancia de deícticos para crear un diálogo con el receptor del mensaje. En la canción de la edición 16 del año $2009^{31}$ se puede observar el propósito principal de la Korrika, unirse en la carrera para el apoyo del idioma independientemente de la procedencia:

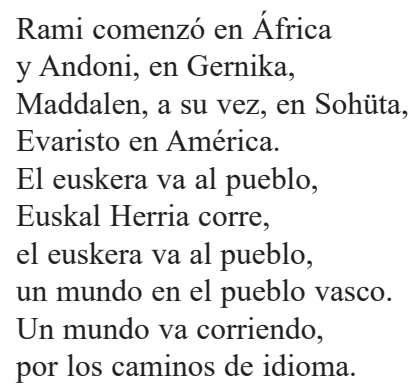

Mantener el ritmo de la carrera y correr por la lengua es posible también gracias a los gritos que se oyen en la calle durante la Korrika, entre los cuales podemos mencionar: Tipi tapa tipi tapa, Korrika! (denominación onomatopéyica para ir paso a paso); (El nombre de un pueblo) ere euskararen alde Pueblo a favor del euskera!; Ika, ika, ika hemen dator Korrika - Ika, ika, ika

${ }^{29}$ Folleto de la Korrika 20 (2017), op. cit.

${ }^{30}$ Korrika 20, en: korrika.eus, <https://www.korrika.eus/cas/abestia-2/zirkorrika-letra-y-fichatécnica.html>, 27 de abril de 2018.

${ }^{31}$ Korrika 16 (Betagarri), en: Euskal Kantak, <http://euskalkantak.blogspot.com.es/2009/04/ korrika-16-betagarri-letra-osorik.html?m=1>, 27 de abril de 2018. 
aquí viene la Korrika; Gora Korrika! - ¡Viva la Korrika!; Gora euskera eta Korrika! - ¡Arriba el euskera y la Korrika!

La transmisión oral de los mensajes se desenvuelve a través del testigo de la Korrika que portan los corredores. Se trata de un palo de madera tallada con una pequeña ikurriña que se hace pasar de mano a mano y que en su interior tiene un mensaje especial que gira en torno al lema y está escrito por un destacado pensador o escritor vasco (en 2017 lo escribió el escritor Joseba Sarrionandia). Dicho mensaje no se hace público hasta el último momento y se lee al finalizar la carrera. De esta forma un papel escrito por una persona de importancia excepcional en el mundo vascoparlante recorre todo el territorio en las manos de miles de personas y simboliza el final. La fuerza del pueblo, de cada participante, se esconde simbólicamente en este objeto de madera que es una muestra de la continuidad de la transmisión lingüística en el espacio. Si hablamos de la tradición oral en la transmisión de la lengua, necesitamos subrayar que todo el territorio por donde pasa la Korrika durante este periodo de tiempo se convierte en un ambiente de habla vasca: desde el precalentamiento antes de la carrera hasta la lectura del mensaje escondido en el testigo.

\section{La Korrika dentro y fuera de Euskal Herria}

La Korrika es hoy día un referente ineludible de la actividad favorable a la lengua vasca, de la cultura vasca y del euskera en sí; y, además, se ha exportado con éxito a otros países ${ }^{32}$. Es un acontecimiento de importancia tanto a nivel nacional como internacional. La expansión de la Korrika va más allá del territorio de la Comunidad Autónoma Vasca, España y Francia, y adquiere su realización en la diáspora, con la celebración de la Korrika en los cinco continentes. De esta manera, la edición 17 llegó por primera vez a Sydney y a Berlín. El año 2017 gozó con la participación de más de 20 países.

También ha echado raíces en otras comunidades autónomas de España y en otros países de Europa. Ha servido como fuente de inspiración para los defensores del catalán, que organizan la Correlengua en favor del catalán desde el año 1993; en Galicia Correlingua apoya el gallego desde el año 2000. También existen las carreras en favor del bretón, $A r$ Redadeg, iniciada en 2008; Rith en Irlanda (desde el 2010), y Ras yr laith en Gales (desde el 2014).

No existe un perfil típico de la gente que participa en las carreras, dado que la continuidad del evento se realiza a través de la participación de varias generaciones, de niños a ancianos, de clases sociales (peñas de colegas, alcaldes, alumnos y maestros) y géneros. Así, en la Korrika de 2017 en Navarra participaron representantes del Gobierno Vasco (viceconsejera de Política Lingüística M. Dobaran), la presidenta del Parlamento Vasco (B. Tejería), los

${ }^{32}$ Folleto de la Korrika 20 (2017), op. cit. 
alcaldes de Vitoria, Pamplona, Bilbao y San Sebastián, los responsables del Departamento de Salud y de Osakidetza para llamar atención sobre el uso del euskera en el sector de la sanidad. A la hora de caracterizar la importancia del fútbol a principios del siglo XX, Pujadas afirma que a su valoración como expresión de la cultura y la modernidad se le suma que se convierte en una pasión anclada en la identidad; al mismo tiempo, se quiebra la selección social de sus protagonistas, incorporándose nuevos colectivos ${ }^{33}$. En la Korrika vemos algo parecido: el hecho de que no solo las masas sino también la administración y los directivos participen en la carrera subraya su importancia en todo el territorio de Euskal Herria.

Junto a la Korrika, donde participan representantes de todas las edades, conviene mencionar las Korrika Txikia, cuando profesores, maestros y alumnos corren un día antes o después de la Korrika principal, debido a que muchas veces la oficial tiene lugar a horas poco aptas para los niños: Txikien Korrika $e z$ da txikia - La Korrika de los pequeños no es pequeña ${ }^{34}$. Cada localidad organiza la Korrika Txikia entre las diferentes escuelas con el fin de dar protagonismo a los más pequeños e inculcar la transmisión de la Korrika. El carácter de evento masivo y plural imposibilita cuantificar la cantidad exacta de los participantes.

\section{Resumen} barria:

A modo de resumen, nos gustaría hacer referencia a las palabras de M. Etxe-

En situaciones sociológicas determinadas, caracterizadas por una ruptura irreversible de la comunicación tradicional, el futuro de una lengua minoritaria depende de la posibilidad de provocar un impulso de valoración sobre sí misma y de cómo pueden las lenguas responder a estas exigencias ${ }^{35}$.

El carácter aglutinador de la Korrika ha creado un espacio social con características y funciones muy peculiares que tiene una naturaleza pragmática ambigua: llamar la atención, popularizar el idioma y recabar fondos para su difusión. Se ha convertido en un acontecimiento que moviliza a toda la sociedad de Euskal Herria y sirve como una muestra de la relación que existe entre el deporte (una carrera) y la lengua (el euskera) en el marco del discurso del deporte. La participación aumenta en cada edición y miles de personas trabajan voluntariamente en su organización a través de los comités que se forman en los pueblos y barrios. Durante la carrera propiamente dicha se

\footnotetext{
33 X. Pujadas, op. cit., p. 81.

34 T. del Valle, op. cit., p. 42.

35 M.A. Etxebarria, op. cit., pp. 21-22.
} 
organizan cientos de actividades culturales y lúdicas ${ }^{36}$. Según la perspectiva actual, tiene futuro como herramienta de protección del euskera. La lucha por la gratuidad de la enseñanza del euskera en todo el territorio vascoparlante, la implicación popular de la sociedad se ve como la aspiración más importante para el futuro. Desde el punto de vista del análisis discursivo, la Korrika es un fenómeno abierto, orientado a la acción, dialogal con estructura lineal, inmediatez comunicativa, comunicación natural y con un anclaje en el contexto. De esta manera se puede afirmar con toda certeza que el deporte no deja de ejercer su papel social.

\section{Referencias bibliográficas}

BARTHES Roland

2007 What is Sport?, New Haven, Yale University Press.

CHARAUDEAU Patrick, MAINGUENEAU Dominique

2005 Diccionario de análisis de discurso, Buenos Aires-Madrid, Amorrortu SL.

CONNOR Steven

2011 A Philosophy of Sport, London, Reaktion Books.

ETXEBARRIA AROSTEGUI Maitena

2002 La diversidad de las lenguas en España, Madrid, S.L.U. Espasa Libros. FERRANDO GARCÍA Manuel

2006 Postmodernidad y Deporte: entre la individualización y la masificación. Encuesta sobre hábitos deportivos de los españoles, Madrid, CIS (Centro de Investigaciones Sociológicas).

MAINGUENEAU Dominique

1999 Términos clave para el análisis del discurso, Buenos Aires, Nueva Visión.

OLABUÉNAGA RUIZ José Ignacio

1984 Atlas lingüistico vasco, Gobierno vasco. Presidencia, Gabinete de prospección sociológica. PARBELAS Pierre

2003 Elementos de la sociología del deporte, Málaga, Instituto Andaluz del Deporte, no. 40. PUJADAS Xavier

2011 Atletas y ciudadanos. Historia social del deporte en España 1870-2010, Madrid, Alianza Editorial.

SHI-XU

2005 A cultural approach to discourse, New York, Palgrave Macmillan.

VALLE Teresa del

1988 Korrika: rituales de la lengua en el espacio, Barcelona, Anthropos. ZUBIAGA Félix

2005 Vascuence, inconsciente colectivo, Amorebieta, Erroteta.

\section{Páginas web}

ALKORTA Asier

“Korrika, 1980. Urtetik pausoak ematen", en: Hitza.eus, 11.11.2016, <http://lea-artibaietamutriku.hitza. eus/2016/11/11/Korrika-1980-urtetik-pausoak-ematen/>, 27 de abril de 2018.

${ }^{36}$ Folleto de la Korrika 20 (2017), op. cit. 
BOLETÍN OFICIAL DEL ESTADO

Constitución española, en: BOE <https://www.boe.es/buscar/doc.php?id=BOE-A-1978-31229>, 27 de abril de 2018.

COUNCIL OF EUROPE

European Charter for Regional or Minority Languages (Spanish Version), Strasbourg, 5.11.92, $<$ https://www.coe.int/t/dg4/education/minlang/textcharter/Charter/Charter_es.pdf $>, 27$ de abril de 2018.

DIARIO DIGITAL DE GETXO

"La Korrika llega a Getxo en forma de homenaje a presos etarras", en: Diario digital de Getxo, 23.03.2015, <http://www.contrapuntolocal.es/?p=11156>, 27 de abril de 2018.

\section{DIARIO VASCO}

"Hay muchas maneras de ser "euskalakari",, Diario Vasco, 18.04.2011<http://www.diariovasco. com/ v/20110418/al-dia-local/muchas-maneras-euskalakari-20110418.html>, 27 de abril de 2018.

"Eibarren ere Korrika", Diario Vasco, 23.03.2015, <http://www.diariovasco.com/fotos/sociedad/ 201503/23/eibarren-Korrika-30102864028232-mm.html>, 27 de abril de 2018.

EUSKAL KANTAK

Korrika 16 (Betagarri) letra, en: Euskal Kantak, http://euskalkantak.blogspot.com.es/?m=1, 27 de abril de 2018.

\section{KORRIKA}

Página oficial de la Korrika: <http://www.Korrika.eus/cas>.

\section{NOTICIAS DE NAVARRA}

"Emotivo y simbólico inicio de la Korrika, que este viernes llega a Navarra", Noticias de Navarra, 31.03.2017, <http://www.noticiasdenavarra.com/2017/03/31/sociedad/navarra/emotivo-ysimbolico-arranque-de-la-Korrika-que-hoy-llega-a-navarra>, 27 de abril de 2018.

"Iruña recibe a la Korrika", Noticias de Navarra, 9.04.2017<http://www.noticiasdenavarra. com/2017/ 04/09/sociedad/navarra/iruna-esta-lista-para-recibir-a-la-Korrika>, 27 de abril de 2018.

NAVARRA.COM

„La Korrika también sirvió para homenajear a los detenidos por los graves incidentes en Pamplona”, en: Navarra.com, 3.04.2017, <https://navarra.elespanol.com/articulo/sociedad/korrikadetenidos-graves-incidentes-pamplona-homenaje-sucesos/20170403080459106591.html> 27 de abril de 2018 .

\section{To run the language: The phenomenon of Korrika}

Keywords: minority languages — sports discourse - euskera — Korrika — slogan.

\section{Abstract}

The article deals with the peculiarities of the phenomenon of modern Spanish sports discourse, which is studied as an entity clearly defined by sociocultural peculiarities. Particularly, it highlights the modern Basque linguistic situation and its minority language position. We describe Korrika, which is a race organised in the Basque Country with the objective of promoting awareness in favour of the Basque language and raising funds to support it. This study shows the connection between language and sports as social phenomena. The article describes the topics and significance of Korrika, the methods of its physical realisation in space, and its main themes. We also give examples of the slogans of different editions, mention the participants' profile and highlight its multidimensional nature in terms of traditional Basque cultural events taking place during the days 
of the race. It may be deduced that mass participation in this social event as well as its unique nature proves that nowadays Korrika has become one of the most important tools in the preservation of the Basque language.

Fecha de recepción: 8.05.2018

Fecha de aceptación: 9.10.2018 